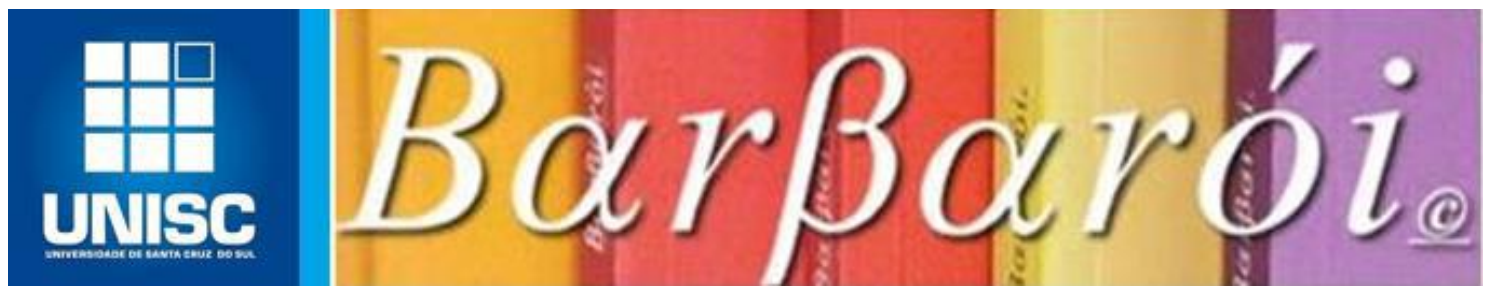

\title{
A QUALIDADE DE VIDA DOS PROFISSIONAIS DA ENFERMAGEM QUE ATUAM NO CENTRO CIRÚRGICO
}

\author{
DOI: http://dx.doi.org/10.17058/barbaroi.v1i53.12986
}

\author{
$*$ \\ Maurício da Silva Roxkow Fraga \\ Rede de Saúde Divina Providência - RSDP - Brasil \\ Prisla Ücker Calvetti \\ Universidade Lasalle - UNILASALLE - Brasil \\ Alexandre Ramos Lazzarotto \\ Universidade La Salle - UNILASALLE - Brasil
}

\section{Resumo}

Este artigo tem por objetivo identificar os domínios da qualidade de vida dos profissionais de enfermagem que atuam nos centros cirúrgicos de quatro hospitais do Rio Grande do Sul. Tratase de um estudo transversal realizado a partir de dois questionários: um sócio demográfico e o SF-36 (Medical Outcomes Study 36 - Item Short - Form Health Survey). Para o tratamento estatístico foram aplicadas frequência, média e desvio padrão em variáveis nominais e o teste $t$ para amostras independentes. A amostra do estudo (125 participantes: $81,6 \%$ sexo feminino) correspondeu a $57,33 \%$ da população total, destes $48,8 \%$ eram do bloco cirúrgico, $30,4 \%$ da central de materiais e $20,8 \%$ da sala de recuperação anestésica. A média de idade foi de 35,8 anos e a média de atuação na instituição foi de 4,6 anos. Os oito domínios avaliados evidenciaram um escore médio superior a 50, porém, "Estado Geral de Saúde", "Dor" e "Vitalidade" apresentaram os menores escores. O estudo revela aspectos importantes sobre a qualidade de vida dos profissionais da enfermagem, apontando fatores com possibilidades de melhorias nos domínios avaliados.

Palavras-chaves: qualidade de vida, centro cirúrgico, enfermagem.

\section{Introdução}

A Organização Mundial da Saúde (OMS) conceitua a qualidade de vida (QV) como a percepção que um indivíduo tem sobre a sua posição na vida em contextos como, cultura, valores, objetivos, expectativas, padrões e preocupações (TANCREDI; BARRIOS; 
FERREIRA, 1998). A QV indica o nível das condições básicas e suplementares do ser humano, as quais envolvem aspectos individuais (bem-estar físico, mental, psicológico e emocional) e coletivos (relacionamentos, família, saúde e trabalho) que afetam a vida humana (OLER; JESUS, 2005).

A partir deste entendimento, pode-se pensar fatores ambientais, como no contexto de trabalho, dentre as possíveis condições de proteção e de risco do bem-estar naquele ambiente no qual se exerce uma função (EFROM; MAGNAN, 2018). Na área da saúde, profissionais que atuam em instituições hospitalares que, convivem com o adoecimento e a morte de pacientes, e lidam com os parentes de pacientes internados, enfrentam, no seu cotidiano, situações que impactam na sua qualidade de vida (EFROM; MAGNAN, 2018).

Neste contexto, destaca-se o centro cirúrgico (CC) como uma unidade onde os profissionais convivem com aspectos que os desafiam diariamente, tais como: intervenções de alto risco; atendimento à trauma; e situações de urgência. Estes fatores, associados ao estresse cotidiano e às longas jornadas de trabalho, influenciam na segurança e no bem-estar desses profissionais (AORN, 2002; SILVA; RODRIGUES; CESARETTI, 1997; GIACOMONI, 2018).

Uma revisão da literatura realizada pelos autores deste estudo, efetuada entre o período de janeiro de 2010 e maio de 2018, nas bases de dados SciELO e PubMed, sobre o tema "qualidade de vida", evidenciou que a expressiva maioria de estudos foram desenvolvidos com portadores de doenças crônicas. Sendo assim, faz-se necessário o desenvolvimento de estudos que visem à saúde do trabalhador da saúde, no intuito de identificar e analisar possíveis mecanismos e organizações implicados no processo saúde e doença no trabalho, como, por exemplo, ambulatórios e hospitais.

Considerando o ambiente do $\mathrm{CC}$ e a lacuna de estudos realizados sobre a QV dos profissionais que nele atuam, elaborou-se um estudo com o objetivo analisar os domínios da qualidade de vida dos profissionais de enfermagem que atuam nos centros cirúrgicos de quatro hospitais do estado do Rio Grande do Sul.

\section{Método}

Trata-se de um estudo transversal analítico. O projeto de pesquisa obteve a aprovação do Comitê de Ética em Pesquisa da Universidade La Salle (CAAE 71371217.0.0000.5307). Como critério de inclusão, convidou-se enfermeiros e técnicos em enfermagem que possuíssem vínculo empregatício com um dos quatro hospitais do estado do Rio Grande do Sul e que 
atuassem no bloco cirúrgico, na sala de recuperação anestésica, na central de materiais e na esterilização. A partir do cálculo amostral obteve-se uma amostra de 125 participantes.

Para a coleta dos dados foram utilizados dois questionários: um para conhecer o perfil profissional dos participantes e o outro para identificar o escore da QV dos mesmos. O instrumento utilizado para avaliar a QV foi o SF-36 (Medical Outcomes Study 36 - Item Short - Form Health Survey), formado por 36 itens sobre os hábitos de vida organizados em oito domínios: capacidade funcional, aspectos emocionais, aspectos físicos, saúde mental, aspectos sociais, dor, vitalidade e estado geral de saúde. Avaliou-se os domínios de forma independente, com um escore final do estado de saúde que variou de 0 a 100 (CICONELLI, 1997).

A coleta dos dados foi realizada de acordo com as seguintes etapas: a)explicação da pesquisa, convite e assinatura do Termo de Consentimento Livre e Esclarecido, b)explicação e preenchimento dos questionários, c)depósito dos questionários na urna situada no CC. Ressaltase que os dois questionários foram preenchidos anonimamente e depositados na urna de material resistente, lacrada e com paredes de cor sólida (proporcionado maior segurança ao participante) que ficou no CC durante o período da coleta dos dados.

Os resultados das variáveis nominais foram expressos em frequência e os resultados das variáveis contínuas em média e desvio padrão. Pelo teste de Kolmogorov-Smirnov verificouse a normalidade dos dados. Para a comparação entre os domínios com as variáveis categoria profissional, sexo e jornada dupla de trabalho, foi utilizado o teste $t$ para amostras independentes. Os dados foram analisados no programa Statistical Package for Social Sciences (SPSS), versão $21.0(\mathrm{p}<0,05)$.

\section{Resultados e discussão}

Para a realização deste estudo foram selecionados dois perfis de profissionais, sendo que ambos atuam em conjunto, porém, exercem funções com responsabilidades distintas. No que concerne ao $\mathrm{CC}$, o ambiente de trabalho está diretamente associado à $\mathrm{QV}$ do individuo em diferentes níveis ou funções. $\mathrm{Na}$ busca por melhor QV, os principais fatores para os melhores resultados estão associados ao quanto o indivíduo recebe de atendimento às suas expectativas (EFROM; MAGNAN, 2018).

A amostra do estudo (125 participantes: $81,6 \%$ do sexo feminino) correspondeu a $57,33 \%$ da população das unidades do CC dos hospitais, estratificada em bloco cirúrgico $(48,8 \%)$ central de materiais e esterilização $(30,4 \%)$ e sala de recuperação anestésica $(20,8 \%)$. A equipe de profissionais de enfermagem era composta por $20 \%$ enfermeiros e $80 \%$ de técnicos 
em enfermagem. A média de idade foi de 35,8 anos, com uma média de 4,6 anos de atuação na respectiva instituição. De acordo com os resultados de Paschoal, Zanier e Whitaker (2007), a média de idade dos profissionais que atuam em centro cirúrgico é entre 35 e 49 anos, assim como, o tempo de atuação profissional varia entre 1 e 5 anos (OLER e JESUS, 2005).

$\mathrm{Na}$ Tabela 1 está descrito o perfil profissional da enfermagem do $\mathrm{CC}$ dos quatro hospitais.

Tabela 1: Perfil profissional dos participantes do estudo $(n=125)$

\begin{tabular}{lc}
\hline Variáveis & $\mathrm{n}=125$ \\
\hline Categoria Profissional & $100(80 \%)$ \\
Técnico (a) em enfermagem & $25(20 \%)$ \\
Enfermeiro & \\
Setor de atuação & $61(48,8 \%)$ \\
Bloco Cirúrgico & $38(30,4 \%)$ \\
Central de Materiais e Esterilização & $26(20,8 \%)$ \\
Sala de Recuperação Anestésica & $5,11(4,52)$ \\
Tempo de Atuação no setor (a) & \\
Sexo & $102(81,6 \%)$ \\
Feminino & $23(18,4 \%)$ \\
Masculino & $35,80(6,38)$ \\
Idade & $10,08(5,65)$ \\
Tempo de atuação profissional (em anos) (b) & $4,68(3,55)$ \\
Tempo de atuação na instituição: (em anos) (c) & \\
Jornada Múltipla de trabalho & $88(70,4 \%)$ \\
Não & $37(29,6 \%)$ \\
Sim &
\end{tabular}

Na Tabela 2 estão descritas as análises descritivas estratificadas por domínios da QV dos participantes do estudo.

Tabela 2: Escores dos domínios da qualidade de vida $(\mathbf{n}=125)$

\begin{tabular}{lcccc}
\hline Domínios & Mínimo & Máximo & Média & DP \\
\hline Capacidade Funcional & 50 & 100 & 86,24 & 13,086 \\
Aspectos Emocionais & 0 & 267 & 83,74 & 35,867 \\
Aspectos Físicos & 0 & 100 & 82,6 & 27,169 \\
Saúde Mental & 28 & 100 & 74,72 & 15,728 \\
Aspectos Sociais & -13 & 100 & 72,76 & 24,703 \\
Dor & 2 & 100 & 65,07 & 21,741 \\
Vitalidade & 5 & 90 & 63,04 & 18,739 \\
Estado Geral de Saúde & 27 & 87 & 59,51 & 11,571 \\
\hline & & & & \\
Escore Total & 28 & 94 & 73,47 & 14,229 \\
\hline \multicolumn{1}{c}{ DP: desvio padrão. } & & & &
\end{tabular}


Conforme os escores obtidos nos oito domínios avaliados pelo estudo, a QV dos profissionais de enfermagem possui um resultado satisfatório, pois de acordo com a sintaxe do instrumento utilizado, todos os domínios atingiram um escore médio superior a 50. Os domínios "Estado Geral de Saúde”, "Dor" e "Vitalidade" apresentaram os menores escores.

A Tabela 3 apresenta a comparação entre a categoria profissional e os escores dos domínios da qualidade de vida.

Tabela 3: Comparação entre a categoria profissional e os escores dos domínios da qualidade de vida $(\mathrm{n}=125)$

\begin{tabular}{lccc}
\hline Domínios & $\begin{array}{c}\text { Enfermeiro } \\
\mathbf{n}=\mathbf{2 5}\end{array}$ & $\begin{array}{c}\text { Técnico de enfermagem } \\
\mathbf{n}=\mathbf{1 0 0}\end{array}$ & $\boldsymbol{p}$ \\
\hline Capacidade Funcional & $87,60(11,64)$ & $85,90(13,45)$ & 0,53 \\
Aspectos Emocionais & $93,36(16,55)$ & $81,83(38,92)$ & $0,02^{*}$ \\
Aspectos físicos & $90(16,13)$ & $80,75(29,05)$ & $0,03^{*}$ \\
Saúde Mental & $80,16(14,52)$ & $73,36(15,77)$ & $0,04^{*}$ \\
Aspectos Sociais & $77,6(23,91)$ & $71,55(24,86)$ & 0,26 \\
Dor & $76,36(19,62)$ & $63(21,84)$ & $0,02^{*}$ \\
Vitalidade & $67,8(20,46)$ & $61,85(18,19)$ & 0,19 \\
Estado Geral de Saúde & $\underline{64,12(12,85)}$ & $\underline{58,36(10,99)}$ & $\underline{0,04^{*}}$ \\
\hline \hline & & & \\
Escore Total & $79,16(10,61)$ & $72,05(14,69)$ & $0,01^{*}$ \\
\hline \hline
\end{tabular}

Resultados expressos em média e desvio padrão.

$* \mathrm{p}<0,05$

A Tabela 4 apresenta a comparação entre o sexo do profissional e os escores dos domínios da QV.

Tabela 4: Comparação entre o sexo do profissional e os escores dos domínios da qualidade de vida $(\mathbf{n}=125)$

\begin{tabular}{lccc}
\hline \multicolumn{1}{c}{ Domínios } & $\begin{array}{c}\text { Masculino } \\
\mathbf{n}=\mathbf{2 3}\end{array}$ & $\begin{array}{c}\text { Sexo } \\
\text { Feminino } \\
\mathbf{n = 1 0 2}\end{array}$ & $\boldsymbol{p}$ \\
\hline Capacidade Funcional & $85,87(14,66)$ & $86,32(12,78)$ & 0,89 \\
Aspectos físicos & $82,61(29,61)$ & $82,60(26,74)$ & 0,99 \\
Dor & $69,57(21,50)$ & $64,06(21,77)$ & 0,27 \\
Estado Geral de Saúde & $61,26(10,15)$ & $59,12(11,87)$ & 0,38 \\
Vitalidade & $59,12(11,87)$ & $60,65(18,98)$ & 0,5 \\
Aspectos Sociais & $69,22(26,36)$ & $73,56(24,37)$ & 0,47 \\
Aspectos Emocionais & $85,52(29,84)$ & $83,33(37,20)$ & 0,76 \\
Saúde Mental & $70,61(14,45)$ & $75,65(15,92)$ & 0,14 \\
\hline \hline & & & \\
Escore total & $73,17(13,55)$ & $73,54(14,40)$ & 0,9 \\
\hline \hline
\end{tabular}

Resultados expressos em média e desvio padrão. 
Em relação à jornada de trabalho, $70,4 \%$ dos participantes não atuavam em dupla jornada e 29,6\% trabalhavam em duas ou mais instituições. Estes percentuais são menores do que aqueles evidenciados no estudo de Oler e Jesus (2005), no qual 47\% dos profissionais atuavam em mais de uma jornada de trabalho.

A Tabela 5 apresenta um comparativo entre a jornada dupla de trabalho e os escores dos domínios da QV.

Tabela 5: Comparação entre jornada dupla de trabalho e os escores dos domínios da qualidade de vida $(\mathrm{n}=125)$

\begin{tabular}{lccc}
\hline Domínios & \multicolumn{3}{c}{$\begin{array}{c}\text { Jornada dupla de trabalho } \\
\text { Não }\end{array}$} \\
& $\mathbf{n = 3 7}$ & $\mathbf{n = 8 8}$ & $\boldsymbol{p}$ \\
\hline Capacidade Funcional & $86,49(14,52)$ & $86,14(12,52)$ & 0,89 \\
Aspectos físicos & $85,14(25,99)$ & $81,53(27,72)$ & 0,49 \\
Dor & $67,24(21,73)$ & $64,16(72,80)$ & 0,47 \\
Estado Geral de Saúde & $53,90(12,11)$ & $59,60(11,40)$ & 0,89 \\
Vitalidade & $64,46(18,92)$ & $62,44(18,73)$ & 0,58 \\
Aspectos Sociais & $74,16(24,27)$ & $72,12(24,99)$ & 0,67 \\
Aspectos Emocionais & $88,30(30,63)$ & $81,82(37,85)$ & 0,31 \\
Saúde Mental & $78,27(14,22)$ & $73,23(16,16)$ & 0,08 \\
\hline \hline & & & \\
Escore total & $75,35(13,22)$ & $72,68(14,32)$ & 0,32 \\
\hline \hline
\end{tabular}

Resultados expressos em média e desvio padrão.

Os domínios "Estado Geral de Saúde", "Dor" e "Vitalidade" apresentaram os menores escores. O domínio "Estado Geral de Saúde” apresentou um escore médio de (média:59,5; desvio padrão:11,57) sendo o domínio mais comprometido entre os pesquisados. De acordo com Oler e Jesus (2005), este domínio avalia o conceito de percepção geral da saúde do individuo e possui, em média, um escore superior (média:79,3; desvio padrão:14,8), considerando não só o estado geral de saúde atual do participante mas também a resistência à doença e a sua percepção sobre aparência saudável (OLER, 2005; REMOR; CASTRO, 2018).

Na percepção do "Estado Geral de Saúde", o técnico em enfermagem indicou um escore mais comprometido (média:58,36; desvio padrão:10,99) quando comparado ao enfermeiro (média:64,12; desvio padrão:12,85), com diferenças significativas $(p=0,04)$, evidenciando a necessidade de atenção dos gestores no desenvolvimento de atividades que sensibilizem os profissionais sobre a sua autoestima e saúde no ambiente de trabalho. Para este domínio, valores baixos indicam que a pessoa considera a sua saúde debilitada e que, provavelmente, piorará. Isto demonstra uma percepção ruim sobre a saúde, bem como fator de risco para baixo 
desempenho profissional (CICONELLI, 1997).

O segundo domínio com menor escore foi a “Dor” (média:65,07; desvio padrão:21,74), que representa a dor física, não só a partir da sua intensidade, mas também por desconforto físico, extensão, forma ou como a dor interfere nas atividades usuais. Uma característica própria do CC é a necessidade constante de esforço físico por parte dos profissionais de ambas as categorias, estando presente o dispêndio elevado de força muscular ou gasto de energia físico, fatores que contribuem para a geração da fadiga, do desconforto e, principalmente, dor (AORN, 2002).

O técnico em enfermagem desempenha um papel direto na assistência ao paciente, diferentemente do enfermeiro, que possui atribuições de gerência e coordenação. O estudo evidenciou um escore mais comprometido para o domínio "Dor" (média:63; desvio padrão:21,84) para o técnico em enfermagem em comparação ao enfermeiro (média:76; desvio padrão:19,62) $(p=0,02)$, indicando a necessidade de maior atenção na prevenção de fatores geradores de desgaste físico para esses no ambiente de trabalho (SILVA; RODRIGUES; CESARETTI, 1997; GIACOMONI, 2018).

O terceiro domínio foi "Vitalidade", que obteve um escore médio (média:63,04; desvio padrão:18,73), sendo que o técnico em enfermagem obteve um escore menor (média:61,85; desvio padrão:18,19), comparando-se ao enfermeiro (média:67,8; desvio padrão:20,46), porém, não houve diferenças significativas $(p=0,19)$ entre as duas categorias. Haddad (2000) afirma que este domínio avalia a percepção do participante sobre seus níveis de energia e fadiga e os escores médios encontrados variam entre 20 e 45 pontos (OLER e JESUS, 2005). Os níveis baixos indicam cansaço ou desmotivação, na maior parte do tempo, o que permite captar melhor as condições e elaborar estratégias para promover bem-estar no ambiente de trabalho (HADDAD, 2000).

Estratégias como observar e coletar sugestões dos profissionais referentes à prática ou sobre possíveis melhorias no ambiente de trabalho podem ser efetivas para gestores que buscam maior rendimento de suas equipes (SOBECC, 2013). Por fim, destaca-se que muitas são as variáveis que podem impactar a QV de profissionais da enfermagem que atuam em ambiente hospitalar em blocos cirúrgicos, como, por exemplo, autorregulação do comportamento em saúde; crenças de saúde e cognições de doenças; personalidade; gênero; apoio social; níveis de estresse; e autocuidado em saúde. 


\title{
Considerações finais
}

A qualidade de vida dos profissionais de enfermagem que atuavam no centro cirúrgico obteve resultado satisfatório, pois todos os domínios atingiram um escore médio superior a 50. Os domínios "Estado Geral de Saúde", "Dor" e "Vitalidade" apresentaram os menores escores. Os enfermeiros obtiveram escores superiores quando comparados aos técnicos em enfermagem em todos os domínios. Considerando as variáveis sexo e atuação em dupla jornada de trabalho, não foram encontradas diferenças significativas.

É relevante a atenção dos gestores sobre fatores que interferem positivamente e que possam facilitar e satisfazer as necessidades do profissional ao desenvolver suas atividades, tanto em relação ao fortalecimento de sua imagem quanto na qualidade ergonômica. Tornamse necessários mais estudos para a avaliação e para o acompanhamento dos índices de QV do trabalhador de enfermagem.

\section{THE QUALITY OF LIFE OF NURSING PROFESSIONALS WORKING IN THE SURGICAL CENTER}

\begin{abstract}
This article aims to identify the domains of the nursing professional's quality of life domains that work in the surgical centers of four hospitals in Rio Grande do Sul. It is a cross-sectional study which has been realized as of two questionnaires, one was a socioeconomic and the other one was the SF-36 (Medical Outcomes Study 36 - Item Short - Form Health Survey). For statistics processing, frequency, mean and standard deviation have been applied to nominal variables and $\mathrm{T}$ Test for independent samples. The sample of the study (125 participants: 81,6\% female gender) have corresponded to $57,33 \%$ of the total population, from that total $48,8 \%$ are from the surgical center, $30,4 \%$ are from the material center, and $20,8 \%$ are from the anesthesia recovery room. The average age is of 35,8 years old and the mean of working years in the institution is of 4,6 years. The eight domains which have been evaluated, have obtained average score superior to 50,0, although, "General State of Health", "Pain" and "Vitality" have presented the minor scores. The study discloses important aspects regarding the interference in the professionals' quality of life, pointing out factors with potential for improvement among the evaluated domains.
\end{abstract}

Keywords: quality of life, surgical center, nursing.

\section{REFERÊNCIAS}

ASSOCIAÇÃO BRASILEIRA DE ENFERMEIROS DE CENTRO CIRÚRGICO RECUPERAÇÃO ANESTÉSICA E CENTRO DE MATERIAL E ESTERILIZAÇÃO SOBECC. Manual. 6. ed. São Paulo: Manole, 2013.

ASSOCIATION OF PERIOPERATIVE REGISTERED NURSES - AORN. Standards, recommended practices, guidelines. Denver: AORN, 2002. 
CICONELLI, R. Tradução para o português e validação do questionário genérico de avaliação de qualidade de vida: Medical Outcomes Study 36. 1997. P. 14-36. Universidade Federal de São Paulo, 1997.

EFROM, C.; MAGNAN, E. A saúde do trabalhador na perspectiva da psicologia positiva organizacional e do trabalho. In: VAZQUEZ, A.; HUTZ, C. (Org.). Aplicações da Psicologia Positiva: trabalho e organizações. São Paulo: Hogrefe, 2018. p. 63-82.

HADDAD, M. Qualidade de vida dos profissionais de enfermagem. Revista Espaço Saúde, Londrina, v. 1, n. 2, p. 75-88, 2000.

OLER, F.; JESUS, A. Qualidade de vida dos profissionais do centro cirúrgico. Revista Arquivos de Ciências da Saúde, São José do Rio Preto, v. 12, n. 2, p. 102-10, 2005.

PASCHOAL S.; ZANIER, S.; WHITAKER I. Y. Qualidade de vida dos trabalhadores de enfermagem de unidades de terapia intensiva. Revista ACTA Paulista de Enfermagem, São Paulo, v. 20, n. 3, p.305-10, 2007.

REMOR, E.; CASTRO, E. Bases teóricas na aplicação da Psicologia da Saúde. In: Elisa Kern de castro e Eduardo Remor. Bases teóricas da Psicologia da Saúde. Curitiba: Appris, 2018. p. 231-245.

ROSANA, M. Tradução para a língua portuguesa e validação do questionário genérico de avaliação de qualidade de vida SF-36. Revista Brasileira de Reumatologia, São Paulo, v. 39, n.3, p. 31-74, 1999.

GIACOMONI, C. H.; HUTZ, C. S. Psicologia Positiva: uma nova perspectiva em saúde. In: Ana Cristina Garcia Dias. (Org.). Psicologia e Saúde: Pesquisas e Reflexões. Santa Maria: Editora da UFSM, 2009, p. 161-175.

SILVA, M.; RODRIGUES, A.; CESARETTI, I. Reflexões: a ética no exercício da enfermagem em centro cirúrgico. In:Maria D'Ápparecida Andrade Silva. Enfermagem na unidade de centro cirúrgico. 6. ed. São Paulo: EPU, 1997. p.76-167.

TANCREDI, F.; BARRIOS, S.; FERREIRA, J. Planejamento em saúde. In: Gonzalo Vecina Neto, Valéria Terra, Raul Cutait e Luiz Eduardo C. Junqueira Machado. Saúde \& Cidadania, São Paulo: Faculdade de Saúde Pública, 1998. p.11-26.

Data de recebimento: $24 / 12 / 2018$

Data de aceite: 04/09/2019

\section{Sobre os autores:}

Maurício da Silva Roxkow Fraga é pós-graduado Lato Sensu em Centro Cirúrgico, Sala de Recuperação e Central de Materiais (Hospital Moinhos de Vento) e Mestrando em Saúde e Desenvolvimento Humano na Unilasalle. Endereço eletrônico: mauricio_fraga@hotmail.com

Prisla Ücker Calvetti é Doutora em Psicologia, na área de Psicologia Clínica, pela Pontifícia Universidade Católica do Rio Grande do Sul e Pós-doutorado pela Universidade Federal do Rio 
Grande do Sul - UFRGS. Endereço eletrônico: prisla.calvetti@gmail.com

Alexandre Ramos Lazzarotto é Doutor em Ciências do Movimento Humano pela Universidade Federal do Rio Grande do Sul. Endereço eletrônico: alazzar@terra.com.br 